\title{
Molecular dynamics of iso-amyl bromide by dielectric spectroscopy, and the effects of a nonpolar solvent, 2-methylpentane, on the spectral features
}

\author{
O. E. Kalinovskaya and J. K. Vij ${ }^{\text {a }}$ \\ Laboratory of Advanced Materials, Department of Electronic and Electrical Engineering, Trinity College, \\ Dublin 2, Ireland
}

(Received 13 July 1999; accepted 28 September 1999)

\begin{abstract}
To gain insight into the effects of the weakening of the electrostatic interactions on molecular dynamics when polar molecules are dissolved in a nonpolar solvent, the dielectric polarization and relaxation behaviors of iso-amylbromide and its $50 \mathrm{~mol} \%$ solution in 2-methylpentane have been studied in detail over the frequency range, $1 \mathrm{mHz}-1 \mathrm{MHz}$, and a temperature range approaching their liquid to glass transition. Features of the (i) $\alpha$-relaxation spectrum, (ii) the Johari-Goldstein relaxation process in the liquid state at low temperatures, with an asymmetric spectral shape, and (iii) the temperature dependence of the relaxation dynamics have been determined and the effects of weakening of the electrostatic interaction on these features examined. The high-frequency wing of the loss spectrum of the $\alpha$-relaxation is proportional to $\omega^{-\beta}$. The dynamics of its $\alpha$-relaxation follows the Arrhenius equation initially at high temperatures and thereafter the Vogel-FulcherTamman equation. Alternative equations for the change in the relaxation rate have been discussed. A decrease in the dipole-dipole interaction and reduction in the internal field in a solution with a nonpolar solvent leads to a remarkable change in the shape of the relaxation spectra at high frequencies such that the dielectric loss for the $\alpha$-relaxation becomes proportional to $\omega^{-\alpha \beta}$, with $\alpha$, $\beta<1$. The relaxation spectra of iso-amyl bromide dissolved in 2-methylpentane follows the $\mathrm{H}-\mathrm{N}$ function and therefore behaves similar to a polymer, whereas for pure iso-amyl bromide follows the Davidson-Cole behavior. () 1999 American Institute of Physics. [S0021-9606(99)50748-4]
\end{abstract}

\section{INTRODUCTION}

Molecular dynamics of supercooled liquids by dielectric spectroscopy is of much current interest, mainly for the purpose of examining (i) the distribution of relaxation times, ${ }^{1-4}$ (ii) the continuous change from its unimodal distribution to a bimodal distribution of relaxation times on cooling towards its glass-transition temperature, $T_{g},{ }^{5-7}$ and (iii) the temperature dependence of the average relaxation time. ${ }^{8,9}$ The relaxation spectra are currently analyzed ${ }^{1-9}$ in terms of a set of equations that are approximately equivalent to the variation of the dielectric loss with frequency according to a "fractional power-law" at both the low-frequency and high frequency sides of the relaxation peak. These equations are the stretched exponential relaxation function ${ }^{10-12}$ in the time domain, Havriliak-Negami or $\mathrm{H}-\mathrm{N}$ equation ${ }^{13}$ in the frequency domain. The latter relationship is a combination of the Cole-Cole relaxation ${ }^{14,15}$ and Davidson-Cole relaxation ${ }^{16}$ functions. In recent studies, ${ }^{17-21}$ of dielectric relaxation in supercooled liquids, the dielectric spectra of glycerol, which was originally found to have an asymmetric shape and had led to the formalism of the Davidson-Cole relaxation function, ${ }^{16}$ have been described in terms of the stretched exponential, and $\mathrm{H}-\mathrm{N}$ (Ref. 13) equations. As the rate of the high-frequency or the Johari-Goldstein relaxation $^{22,23}$ in the liquid state depends on the manner of

${ }^{\text {a) }}$ Author to whom correspondence should be addressed. the fitting of the low-frequency spectra, this has caused a concern about not only the ambiguity of the results deduced from such analyses but also on the validity of the DavidsonCole formalism ${ }^{16}$ for dielectric relaxation in supercooled liquids. For the general case of $\mathrm{H}-\mathrm{N}$ formalism, for frequencies much lower than the frequency of maximum dielectric loss, the low frequency wing of the loss-spectrum varies as $\omega^{+\alpha}$, whereas for frequencies much greater than the frequency of maximum loss, the high frequency wing of the loss spectrum varies as $\omega^{-\alpha \beta}$. H-N formalism reduced to Davidson-Cole for $\alpha=1$.

One objective of this study was to determine whether other supercooled liquids also showed a dielectric spectral shape corresponding to the stretched exponential or $\mathrm{H}-\mathrm{N}$ equation. The second purpose of this study was to examine how a change in the electrostatic interactions of a dipolar molecule in a nondipolar solvent affects the dipolar relaxation dynamics. Pure iso-amyl bromide and its 2-methylpentane mixture were chosen for this study, mainly because it is the original liquid, devoid of H-bonds, for which a skewed arc shape of the complex-plane plots was observed in the studies by three groups; Glarum ${ }^{24}$ who had studied the relaxation of pure iso-amyl bromide at temperatures above its freezing point, Denny, ${ }^{25}$ who studied it in the supercooled liquid state, and Berberian and $\mathrm{Cole}^{26}$ who subsequently extended these data to lower temperatures by using the transient current method. The dielectric relaxation of its solution in 2-methylpentane was studied by Denny and 
Ring. ${ }^{27}$ In the more detailed study of iso-amyl bromide it was noted that although the spectra had a Davidson-Cole, there were deviations at the high frequency end. Also it was noted that the relaxation time deviated from the VogelFulcher-Tamman equation ${ }^{28-30}$ at temperatures above the freezing point of iso-amyl bromide (Fig. 4 in Ref. 26). We will also discuss an analysis of these features in detail here.

\section{EXPERIMENTAL METHODS}

The dielectric permittivity $\epsilon^{\prime}$ and loss $\epsilon^{\prime \prime}$ were measured over the frequency range, $1 \mathrm{mHz}-1 \mathrm{MHz}$ using a Solartron FRA-1255A, frequency response analyzer equipped with a Chelsea dielectric interface. The dielectric cell used was a miniature, tunable parallel plate capacitor containing 18 plates, with an air capacitance of nominally $16 \mathrm{pF}$. (Note parallel plate capacitors with two plates separated by spacers have been used successfully in this frequency range by several groups.) The cell and the sample's temperature was controlled by keeping it inside a cryostat, model Oxford special Spectrodn20 top loading, and an ITC6 temperature controller both purchased from Oxford Instruments. The temperatures were not programmed. Rather these were set and controlled isothermally at the desired value by its manual setting. The temperature was controlled to within $\pm 0.1 \mathrm{~K}$ over a period of up to $24 \mathrm{~h}$ required for measurements at the lowest temperature in the lowest frequency range. Temperature of the cell was measured using a Keithley 105A multimeter, by immersing a PT 100 class A sensor in the cell containing the liquid. The maximum tolerance at $73 \mathrm{~K}$ is given as $\pm 0.55^{\circ} \mathrm{C}$ which is better than for most thermocouples.

Iso-amyl bromide (GC grade, purity $>96 \%$ ) and 2-methylpentane (purity $>99 \%$ ) were purchased from Aldrich. Iso-amyl bromide was used after purification by fractional distillation, whereas 2-methylpentane was used as such. Their $50 \mathrm{~mol} \%$ mixture were prepared by accurately weighing the appropriate amounts.

\section{RESULTS}

Instead of the plots of $\epsilon^{\prime}$ and $\epsilon^{\prime \prime}$ in a complex plane, we show the $\epsilon^{\prime}$ and $\epsilon^{\prime \prime}$ spectra of iso-amyl bromide at 14 selected temperatures from $110.1 \mathrm{~K}$ to $133.3 \mathrm{~K}$ in Fig. 1. Here the spectra obtained at $131.3 \mathrm{~K}$ and $133.3 \mathrm{~K}$ show a lower value of $\epsilon^{\prime}$ and $\epsilon^{\prime \prime}$ than expected on the basis of the temperature decrease, and the sample was found to have partially crystallized (its freezing point is $161.3 \mathrm{~K}$ ), as was also noted by Denney. ${ }^{25}$ This partial crystallization of iso-amyl bromide does not seem to introduce an additional relaxation process, or shift the peak of the liquid's original relaxation process, introduces only a fractional change in the magnitude of the orientation polarization; the amount of liquid crystallized is $\sim 20 \%$ at $131.3 \mathrm{~K}$ and $60 \%$ at $133.4 \mathrm{~K}$. In both cases the crystallization appeared to be slow enough to alter data during the period of $2 \mathrm{~h}$ for collecting the spectra.

A typical spectra measured at $115.1 \mathrm{~K}$ is shown in Fig. 2. Its shape was analyzed by using the WINDETA program provided by Novocontrol, Hundsagen, Germany. This computational algorithm has been used generally in the analysis of the dielectric data by Fischer and co-workers. The con-

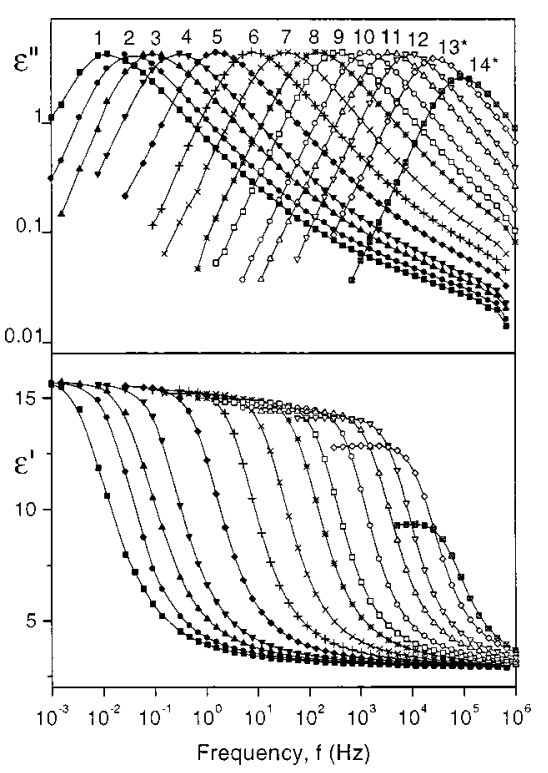

FIG. 1. The $\epsilon^{\prime}$ and $\epsilon^{\prime \prime}$ spectra of pure iso-amyl bromide at several temperatures, as follows: (1) $110.1 \mathrm{~K}$, (2) $111.15 \mathrm{~K}$, (3) $112.1 \mathrm{~K}$, (4) $112.8 \mathrm{~K}$, (5) $115.1 \mathrm{~K}$, (6) $117.2 \mathrm{~K}$, (7) $119.15 \mathrm{~K}$, (8) $121.5 \mathrm{~K}$, (9) $123.0 \mathrm{~K}$, (10) $125.2 \mathrm{~K}$, (11) $127.1 \mathrm{~K},(12) 129.0 \mathrm{~K},(13 *) 131.3 \mathrm{~K},\left(14^{*}\right) 133.3 \mathrm{~K}$. * refers to partially crystallized samples.

tinuous line is the curve calculated with $\alpha=0.98$ and $\beta=0.55$, the equilibrium dielectric permittivity, $\epsilon_{s}=15.54$, the limiting high-frequency permittivity, $\epsilon_{\infty}=3.12$, and the characteristic relaxation time $\tau=0.161 \mathrm{~s}$. As seen in Fig. 2, the measured values of $\epsilon^{\prime}$ and $\epsilon^{\prime \prime}$ are higher than those calculated and therefore there was a need to fit the entire data to a sum of relaxation spectra according to the equation,

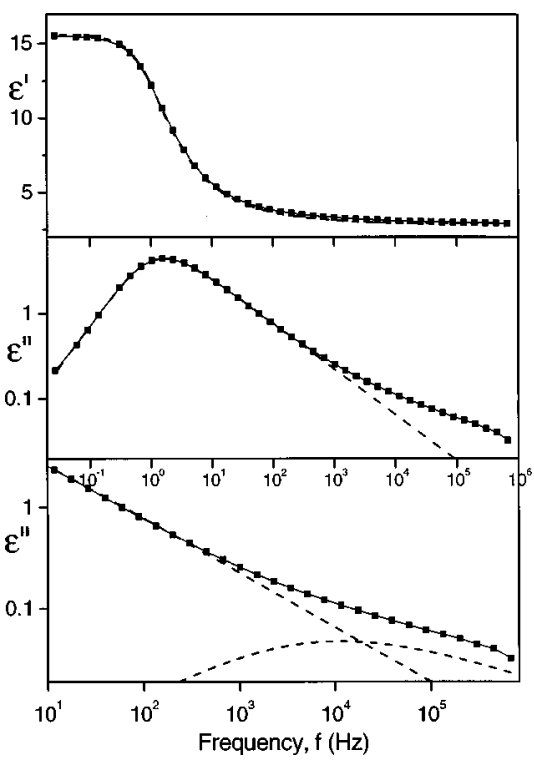

FIG. 2. The $\epsilon^{\prime}$ and $\epsilon^{\prime \prime}$ spectra of pure iso-amyl bromide measured at $115.1 \mathrm{~K}$ is analyzed into one relaxation process, with $\epsilon_{s}=15.54, \epsilon_{\infty}=3.12, f_{m}$ $=1.62 \mathrm{~Hz}, \alpha=0.98$, and $\beta=0.55$. The plot at the bottom shows the resolution of the $\alpha$ - and the Johari-Goldstein relaxation process in isoamylbromide at $115.1 \mathrm{~K}$. The parameters used for the calculations are given in Table I. 
TABLE I. The parameters obtained by an analysis of the dielectric data of iso-amyl bromide according to Eqs. (1) and (2). The symbol * refers to the partially crystallized sample.

\begin{tabular}{|c|c|c|c|c|c|c|c|c|c|c|c|c|}
\hline$T(\mathrm{~K})$ & $\epsilon_{s}$ & $\Delta \epsilon_{1}$ & $\alpha_{1}$ & $\beta_{1}$ & $\tau_{1}(\mathrm{~s})$ & $\Delta \epsilon_{2}$ & $\alpha_{2}$ & $\beta_{2}$ & $\tau_{2}(\mathrm{~s})$ & $f_{m, 1}(\mathrm{~Hz})$ & $f_{m, 2}(\mathrm{~Hz})$ & $\epsilon_{s}-\Sigma \Delta \epsilon_{k}$ \\
\hline 110.1 & 15.8 & 12.84 & 0.94 & 0.54 & 22.59 & 0.22 & 0.77 & 0.35 & $4.43 \times 10^{-3}$ & $1.21 \times 10^{-2}$ & $1.18 \times 10^{2}$ & 2.55 \\
\hline 111.15 & 15.75 & 11.88 & 1 & 0.54 & 7.87 & 0.37 & 0.57 & 0.44 & $6.43 \times 10^{-3}$ & $3.3 \times 10^{-2}$ & $9.46 \times 10^{1}$ & 3.43 \\
\hline 112.1 & 15.71 & 12.54 & 0.99 & 0.50 & 3.17 & 0.3 & 0.49 & 0.43 & $4.57 \times 10^{-4}$ & $8.73 \times 10^{-2}$ & $1.84 \times 10^{3}$ & 2.87 \\
\hline 112.83 & 15.61 & 12.46 & 0.95 & 0.56 & $9.42 \times 10^{-1}$ & 0.32 & 0.54 & 0.53 & $2.92 \times 10^{-4}$ & $2.75 \times 10^{-1}$ & $1.86 \times 10^{3}$ & 2.83 \\
\hline 115.1 & 15.54 & 12.42 & 0.98 & 0.55 & $1.61 \times 10^{-1}$ & 0.26 & 0.74 & 0.60 & $3.44 \times 10^{-5}$ & 1.62 & $1.13 \times 10^{3}$ & 2.86 \\
\hline 117.12 & 15.37 & 12.08 & 0.98 & 0.58 & $3.07 \times 10^{-2}$ & 0.21 & 0.72 & 0.67 & $1.58 \times 10^{-5}$ & 8.09 & $1.65 \times 10^{4}$ & 3.08 \\
\hline 119.15 & 15.2 & 12.43 & 0.95 & 0.61 & $7.09 \times 10^{-3}$ & 0.16 & 0.77 & 1 & $3.06 \times 10^{-6}$ & $3.46 \times 10^{1}$ & $5.21 \times 10^{4}$ & 2.61 \\
\hline 121.49 & 14.99 & 12.22 & 0.96 & 0.60 & $1.67 \times 10^{-3}$ & 0.2 & 0.79 & 0.75 & $2.2 \times 10^{-6}$ & $1.47 \times 10^{2}$ & $1 \times 10^{5}$ & 2.57 \\
\hline 122.99 & 14.81 & 12.07 & 0.98 & 0.58 & $6.65 \times 10^{-4}$ & 0.18 & 0.79 & 0.47 & $1.26 \times 10^{-6}$ & $3.73 \times 10^{2}$ & $2.92 \times 10^{5}$ & 2.56 \\
\hline 125.2 & 14.54 & 11.27 & 1 & 0.61 & $1.77 \times 10^{-4}$ & 0.19 & 0.79 & 0.36 & $9.67 \times 10^{-7}$ & $1.33 \times 10^{3}$ & $5.08 \times 10^{5}$ & 3.08 \\
\hline 127.11 & 14.39 & 11.5 & 0.97 & 0.63 & $6.09 \times 10^{-5}$ & & & & & $3.86 \times 10^{3}$ & & 2.89 \\
\hline 129.04 & 14.09 & 10.83 & 1 & 0.63 & $2.47 \times 10^{-5}$ & & & & & $9.33 \times 10^{3}$ & & 3.26 \\
\hline $131.28 *$ & 12.77 & 9.78 & 1 & 0.63 & $8.02 \times 10^{-6}$ & & & & & $2.86 \times 10^{4}$ & & 2.99 \\
\hline $133.39 *$ & 9.03 & 6.38 & 1 & 0.68 & $2.26 \times 10^{-6}$ & & & & & $9.58 \times 10^{4}$ & & 2.65 \\
\hline
\end{tabular}

$$
\epsilon^{*}(\omega)=\epsilon_{\infty}+\sum_{i=1}^{i=n} \frac{\epsilon_{s, i}-\epsilon_{\infty, i}}{\left[1+\left(i \omega \tau_{i}\right)^{\alpha_{i}}\right]^{\beta_{i}}},
$$

and using the WINDETA algorithm. In Eq. (1) the shape parameters $\alpha_{i}$ and $\beta_{i}$ in Eq. (1) determine the symmetric and asymmetric distribution of relaxation times in the $\epsilon^{\prime}$ and $\epsilon^{\prime \prime}$ spectra. $\alpha_{i}$ and $\alpha_{i} \beta_{i}$ determine the angle of approach towards the limiting low-, and high-frequency values of the dielectric relaxation spectra. An attempt was made to fit the $\epsilon^{\prime}$ and $\epsilon^{\prime \prime}$ spectra to two relaxation regions, i.e., $n=2$.

The analysis of $\epsilon^{\prime}$ and $\epsilon^{\prime \prime}$ spectra thus yielded the shape and relaxation time. A typical analysis of the spectra is also shown in Fig. 2, where the dashed line in the form of an arc shows the calculated spectra at $115.1 \mathrm{~K}$, with the parameters, $\alpha_{2}=0.74, \quad \beta_{2}=0.60, \quad \Delta \epsilon_{2}=0.26, \quad \epsilon_{\infty, 2}=2.86, \quad$ and $\tau_{2}$ $=34.4 \mu \mathrm{s}$.

The various parameters of Eq. (1) at 14 different temperatures thus obtained are listed in Table I. The values of $\alpha_{1}$ and $\beta_{1}$ for the prominent, low-frequency spectra are plotted against the temperature in Fig. 3. The data in Table I and Fig. 3 show that the parameter $\alpha_{1}$ for the prominent relaxation process is $0.98 \pm 0.02$, remaining almost constant with the changing temperature, and $\beta_{1}$ increases from 0.54 at $110.1 \mathrm{~K}$ to 0.61 at $125.2 \mathrm{~K}$.

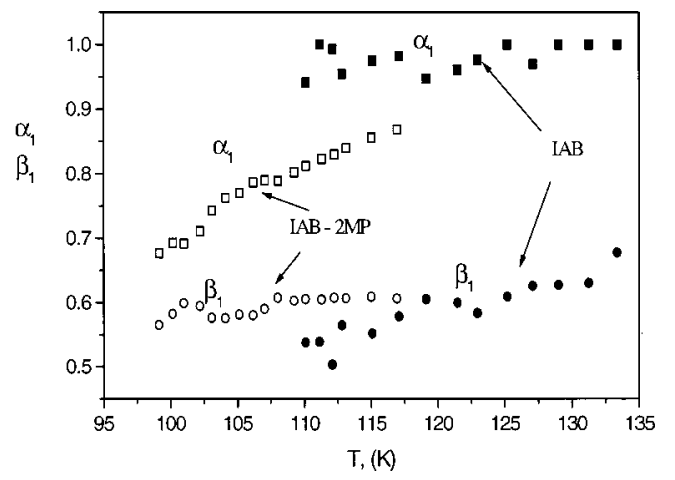

FIG. 3. The plots of the parameters $\alpha$ and $\beta$ against temperature for isoamyl bromide, and $50 \mathrm{~mol} \%$ solution of iso-amyl bromide in 2-methylpentane (both for the main processes). from $^{8}$

The frequency of the relaxation peak was calculated $f_{m, 1}=\left(2 \pi \tau_{1}\right)^{-1}\left(\sin \left[\frac{\alpha_{1} \pi}{2+2 \beta_{1}}\right]\right)^{1 / \alpha_{1}}\left(\sin \left[\frac{\alpha_{1} \beta_{1} \pi}{2+2 \beta_{1}}\right]\right)^{-1 / \alpha_{1}}$.

$f_{m, 1}$ is plotted logarithmically against the reciprocal temperature in Fig. 4. Here $f_{m, 1}$ values calculated from the $\tau$ data provided by Glarum, ${ }^{24}$ Denney, ${ }^{25}$ and Berberian and Cole ${ }^{26}$ are included for comparison. Our results show a similar curve shape, though frequencies of maximum dielectric loss for the low frequency process are somewhat lower than those given in the literature. Frequencies of maximum dielectric loss for the high frequency process are higher. The manner of analysis of the data presented here was not carried out previously for this material, hence there is only a qualitative agreement between our results and those in the literature. The values of $f_{m, 2}$ were calculated from an equation corresponding to Eq. (2) and using the appropriate values of the other parameters. It is plotted also in Fig. 4.

The magnitude of $\boldsymbol{\epsilon}_{s}$ (same as $\boldsymbol{\epsilon}_{s, 1}$ ) is plotted against the temperature in Fig. 5. Here the values are somewhat higher than those reported by others. For example, the earlier value

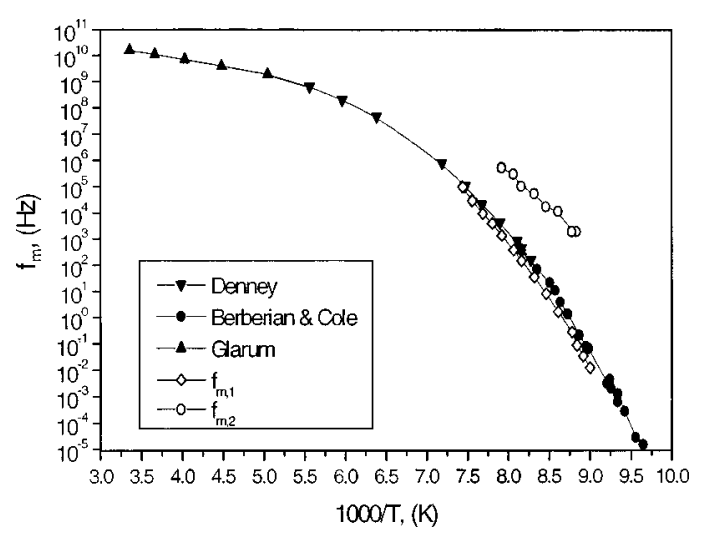

FIG. 4. The plots of $\log \left(f_{m}\right)$ for the $\alpha$-relaxation and the Johari-Goldstein relaxation processes against the reciprocal temperature for pure iso-amyl bromide. Our results are compared with those in the literature, Refs. 25, 26, and 27. 


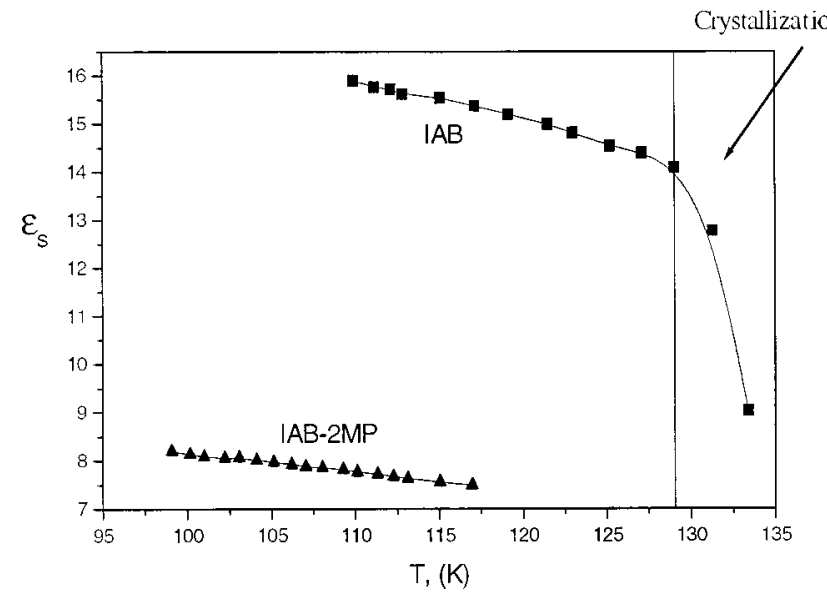

FIG. 5. The $\epsilon_{s}$ of the $\alpha$-relaxation process for pure iso-amyl bromide and its $50 \mathrm{~mol} \%$ solution in 2-methylpentane is plotted against the temperature.

of $\epsilon_{s, 1}$ at $115.7 \mathrm{~K}$ is $13.88,{ }^{26}$ and here at $115.1 \mathrm{~K}$ it is 15.54 . Our values are obtained from the saturated values extrapolated to the lowest frequencies of the measurement, whereas those quoted in the literature are obtained from fits. ${ }^{26}$ In any case, such differences in the values are not unusual and have in fact been found in the various earlier studies of iso-amyl bromide, as discussed by Denney. ${ }^{25}$ These depend on the absolute accuracy of temperature and the purity of the sample.

Similar analysis was carried out for the $\epsilon^{\prime}$ and $\epsilon^{\prime \prime}$ spectra of a $50 \mathrm{~mol} \%$ solution of iso-amyl bromide in 2-methylpentane. Figure 6 shows the $\epsilon^{\prime}$ and $\epsilon^{\prime \prime}$ spectra measured at 18 different temperatures, and Fig. 7 shows the analysis of a typical spectra at $104.1 \mathrm{~K}$, according to Eq. (1). The dashed line is the curve calculated with $\alpha=0.76$ and

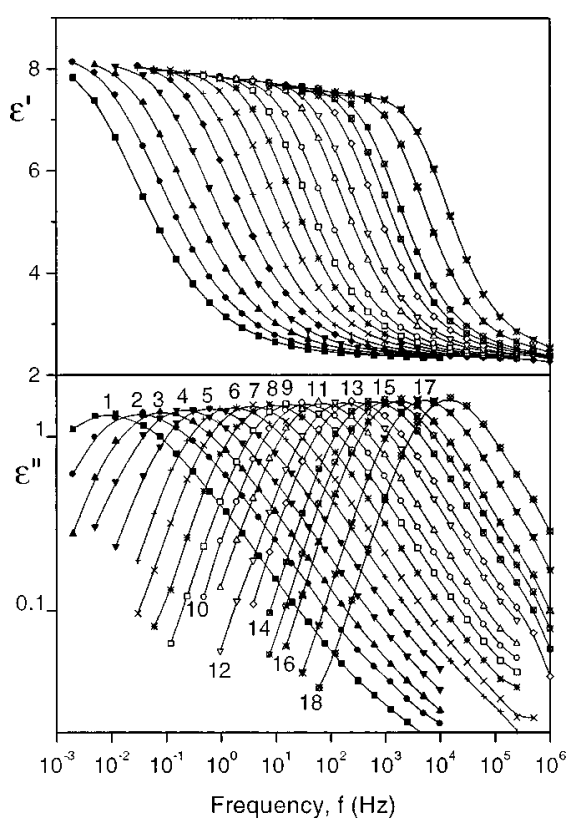

FIG. 6. The $\epsilon^{\prime}$ and $\epsilon^{\prime \prime}$ spectra of $50 \mathrm{~mol} \%$ iso-amyl bromide-2methylpentane mixture at several temperatures, as follows: (1) $98.0 \mathrm{~K},(2)$ 99.2 K, (3) $100.2 \mathrm{~K}$, (4) $101.0 \mathrm{~K}$, (5) $102.2 \mathrm{~K}$, (6) $103.1 \mathrm{~K}$, (7) $104.1 \mathrm{~K}$, (8) 105.1 K, (9) $106.2 \mathrm{~K}$, (10) $107.0 \mathrm{~K}$, (11) $108.0 \mathrm{~K}$, (12) $109.3 \mathrm{~K}$, (13) 110.1 K, (14) $111.3 \mathrm{~K}$, (15) $112.3 \mathrm{~K}$, (16) $113.1 \mathrm{~K}$, (17) $115.0 \mathrm{~K}$, (18) $116.95 \mathrm{~K}$.

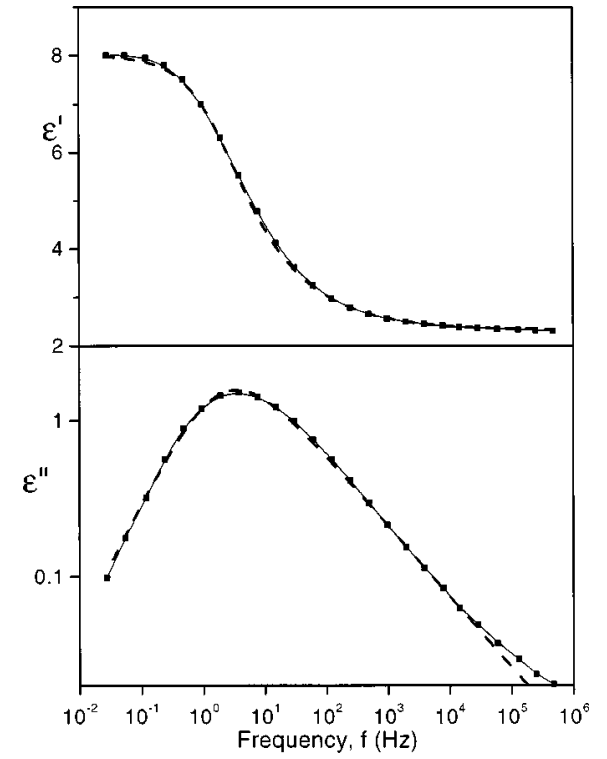

FIG. 7. The $\epsilon^{\prime}$ and $\epsilon^{\prime \prime}$ spectrum of $50 \mathrm{~mol} \%$ solution of iso-amyl bromide in 2-methylpentane measured at $104.1 \mathrm{~K}$ is analyzed by Eq. (1) for one relaxation process with the parameters given in Table II.

$\beta=0.58, \epsilon_{s, 1}=8.01, \epsilon_{\infty, 1}=2.27$, and $\tau_{1}=85.4 \mathrm{~ms}$. Here there is also a relaxation region at high-frequencies, but its relatively small magnitude prevents us from an appropriate analysis. It is conceivable that the high-frequency relaxation would become resolved as a peak at low temperatures in the glassy state, which was not done here. The corresponding parameters for $50 \mathrm{~mol} \%$ solution of iso-amyl bromide in 2-methylpentane are summarized in Table II.

The plot of $\boldsymbol{\epsilon}_{s}$ against the temperature for the $50 \mathrm{~mol} \%$ iso-amyl bromide solution in 2-methylpentane is also shown in Fig. 5, where a comparison against $\epsilon_{s}$ for pure iso-amyl bromide may be made. Our values for $\epsilon_{s}$ are consistently higher than those reported by Denney and Ring. ${ }^{27}$ The values of $f_{m, 1}$ for the solution are plotted against the reciprocal temperature in Fig. 8. The plot is non-Arrhenius and may be

TABLE II. The parameters obtained by an analysis of the dielectric data of $50 \mathrm{~mol} \%$ iso-amyl bromide solution in 2-methylpentane according to Eqs. (1) and (2).

\begin{tabular}{rccccccc}
\hline \hline$T(\mathrm{~K})$ & $\epsilon_{s}$ & $\Delta \epsilon_{1}$ & $\alpha_{1}$ & $\beta_{1}$ & $\tau_{1}(\mathrm{~s})$ & $f_{m, 1}(\mathrm{~Hz})$ & $\epsilon_{s}-\Sigma \Delta \epsilon_{k}$ \\
\hline 99.16 & 8.23 & 6.03 & 0.68 & 0.56 & 11.27 & $3.04 \times 10^{-2}$ & 2.2 \\
100.16 & 8.13 & 5.89 & 0.69 & 0.58 & 3.772 & $8.55 \times 10^{-2}$ & 2.24 \\
101.01 & 8.08 & 5.96 & 0.69 & 0.60 & 1.394 & $2.23 \times 10^{-1}$ & 2.12 \\
102.21 & 8.05 & 5.94 & 0.71 & 0.59 & $5.041 \times 10^{-1}$ & $6.09 \times 10^{-1}$ & 2.11 \\
103.08 & 8.06 & 5.84 & 0.74 & 0.58 & $1.931 \times 10^{-1}$ & 1.60 & 2.22 \\
104.11 & 8.01 & 5.76 & 0.76 & 0.58 & $8.538 \times 10^{-2}$ & 3.53 & 2.27 \\
105.14 & 7.97 & 5.74 & 0.77 & 0.58 & $3.472 \times 10^{-2}$ & 8.53 & 2.23 \\
106.18 & 7.92 & 5.67 & 0.79 & 0.58 & $1.559 \times 10^{-2}$ & $1.87 \times 10^{1}$ & 2.25 \\
107.03 & 7.87 & 5.63 & 0.79 & 0.59 & $8.045 \times 10^{-3}$ & $3.55 \times 10^{1}$ & 2.25 \\
108.03 & 7.85 & 5.60 & 0.79 & 0.61 & $3.996 \times 10^{-3}$ & $6.92 \times 10^{1}$ & 2.25 \\
109.26 & 7.81 & 5.56 & 0.80 & 0.60 & $1.653 \times 10^{-3}$ & $1.67 \times 10^{2}$ & 2.25 \\
110.11 & 7.76 & 5.51 & 0.81 & 0.60 & $9.756 \times 10^{-4}$ & $2.79 \times 10^{2}$ & 2.26 \\
111.32 & 7.72 & 5.47 & 0.82 & 0.60 & $4.364 \times 10^{-4}$ & $6.18 \times 10^{2}$ & 2.26 \\
112.26 & 7.67 & 5.42 & 0.83 & 0.61 & $2.476 \times 10^{-4}$ & $1.08 \times 10^{3}$ & 2.25 \\
113.12 & 7.63 & 5.37 & 0.84 & 0.61 & $1.498 \times 10^{-4}$ & $1.77 \times 10^{3}$ & 2.27 \\
115.02 & 7.55 & 5.27 & 0.86 & 0.61 & $5.142 \times 10^{-5}$ & $5.07 \times 10^{3}$ & 2.28 \\
116.95 & 7.49 & 5.21 & 0.87 & 0.61 & $1.898 \times 10^{-5}$ & $1.36 \times 10^{4}$ & 2.28 \\
\hline \hline
\end{tabular}




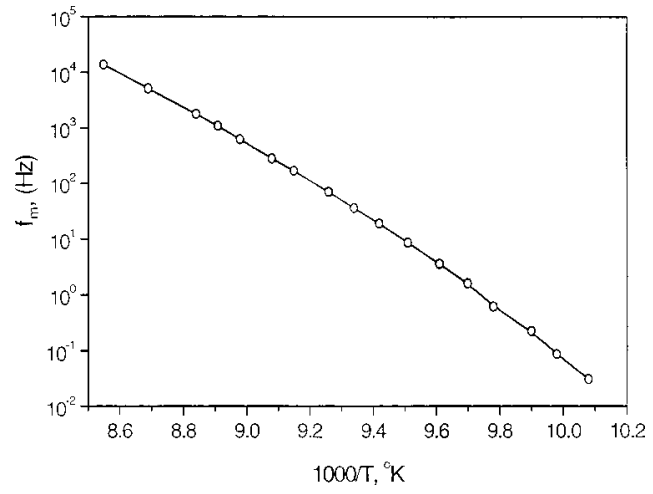

FIG. 8. The plot of $\log (\mathrm{fm})$ for the $\alpha$-relaxation process against the reciprocal temperature for $50 \mathrm{~mol} \%$ iso-amyl bromide solution in 2-methylpentane.

fitted to the Vogel-Fulcher-Tamman equation with parameters, $A=15.17, B=582$, and $T_{0}=64.3 \mathrm{~K}$. Because of the different manner of analysis here to the one used earlier, ${ }^{26} \mathrm{a}$ comparison between the respective data seems unnecessary. Nevertheless, the data were fitted to the various equations in the same manner as for iso-amyl bromide. The parameters obtained are listed in brackets in Table III.

\section{DISCUSSION}

In view of the earlier studies of iso-amyl bromide and its solution in 2-methylpentane, it seems more appropriate to discuss first those features which differ most from the earlier data and conclusions, and then proceed to provide a general discussion of the new features observed here and the effects of the nonpolar solvent on the relaxation spectra.

\section{A. The spectral shape and the effects of the solvent}

In Table I, the observation that the parameter $\alpha$ of Eq. (1), $0.98 \pm 0.02$ close to unity, clearly shows that the relaxation spectra for iso-amyl bromide is of the Davidson-Cole-type ${ }^{16}$ It is very likely that a reduction in $\alpha$ by $\sim 2 \%$ is caused by the effects of dc conductivity and the interfacial polarization as was pointed out by Johari et al. ${ }^{31}$ Nevertheless $\alpha$ is reduced from almost unity to 0.6 for the $50 \%$ isoamyl bromide solution in 2-methyl pentene and its shape seems to fit Eq. (1). In the earlier study, ${ }^{27}$ it was found that none of the (then) three known relaxation functions of the Debye-type, Cole-Cole-type, ${ }^{14,15}$ or DavidsonCole-type ${ }^{16}$ could be fitted to the spectra.

It is evident from Fig. 5 that the parameter $\beta$ does not change when iso-amyl bromide is dissolved in 2-methylpentane, only the parameter $\alpha$, which defines the slope in the low-frequency wing, decreases. This seems to indicate that when internal field effects on the orientation of a dipole are reduced on dissolving a dipolar molecule in a solvent, the relaxation spectral shape is modified.

It may be noted that the increase in the magnitude of relaxation (dielectric amplitude), $\Delta \epsilon$, with temperature, as seen in Tables I and II is more than that expected on the basis of the Curie Law, which requires that $T \Delta \epsilon$ remain constant on cooling, as may also be noted from the data in Table I of Ref. 24. This indicates that there is a substantial dipole-dipole interactions in pure isoamyl bromide, which cause their dipolar alignment in an antiparallel manner. This alignment seems to be considerably reduced, with the net effect that the relaxation spectra of iso-amyl bromide in 2-methaylpentane becomes like that of an amorphous polymer with a broad distribution of relaxation times ${ }^{27}$ and a variety of other molecular liquids. ${ }^{5,8,9}$

As mentioned earlier here, a variety of studies on supercooled liquid glycerol ${ }^{21}$ have found that its relaxation spectra is more appropriately described by the $\mathrm{H}-\mathrm{N}$ equation or by a nonexponential relaxation function, ${ }^{17-20}$ and not by the Davidson-Cole relaxation function. ${ }^{16}$ In view of the fact that the dielectric data on supercooled liquid glycerol had led originally to the discovery of a new relaxation function, it may be worthwhile to re-examine the new data in order to determine whether or not its relatively high dc conductivity and the consequent interfacial polarization (which can be substantial when the surface to volume ratio of the liquid is large) has not contributed significantly to the low-frequency wing of the spectra. Johari and co-workers ${ }^{31,32}$ have recently shown how such contributions may distort the spectral shape and allow it to be fitted by the $\mathrm{H}-\mathrm{N}$ equation.

\section{B. Temperature dependence of the dielectric relaxation time}

The temperature dependence of molecular relaxation time is a feature characteristic of molecular interactions in a liquid, and it varies from a simple Arrhenius to highly nonArrhenius behavior, and there are no theories that can explain this behavior satisfactorily. This has led to a variety of comparative analyses of the plots of $\log \left(f_{m}\right)$ against the reciprocal temperature, the most recent amongst which is one by Stickel et $a l .{ }^{8}$ It seems instructive to follow their manner of analysis here, by using four equations, (i) the Arrhenius equation, (ii) the Vogel-Fulcher-Tamman equation, ${ }^{28-30}$ (iii) the Götze-Sjögren's or the Götze's equation ${ }^{33}$ for the mode coupling theory, and (iv) the Souletic and Bertrand's equation, ${ }^{34}$ all proposed for the temperature dependence of

TABLE III. The values of different parameters obtained by fitting the various equations to the temperature dependence of the relaxation rate for pure iso-amyl bromide (50\% isoamylbromide-pentane mixtures in parentheses).

\begin{tabular}{lllcc}
\hline \hline & \multicolumn{1}{c}{ Function } & \multicolumn{1}{c}{$A$} & \multicolumn{1}{c}{$B, \gamma$} & $T_{0}, T_{c}$ \\
\hline Arrhenius & $\log \left(f_{m}\right)=A-B / T$ & $12.28(31.2)$ & $608(3165)$ & 0 \\
Vogel-Fulcher & $\log \left(f_{m}\right)=A-B /\left(T-T_{0}\right)$ & $16.81(15.17)$ & $787(582)$ & $68.5(64.3)$ \\
Mode-coupling & $\log \left(f_{m}\right)=A+\gamma \cdot \log \left[\left(T-T_{c}\right) / T_{c}\right]$ & $10.05(10.9)$ & $2.82(10.9)$ & $134.7(83.9)$ \\
Souletie & $\log \left(f_{m}\right)=A+\gamma \cdot \log \left[\left(T-T_{c}\right) / T\right]$ & $11.55(17.3)$ & $5.47(17.3)$ & $125.5(80.2)$ \\
\hline \hline
\end{tabular}



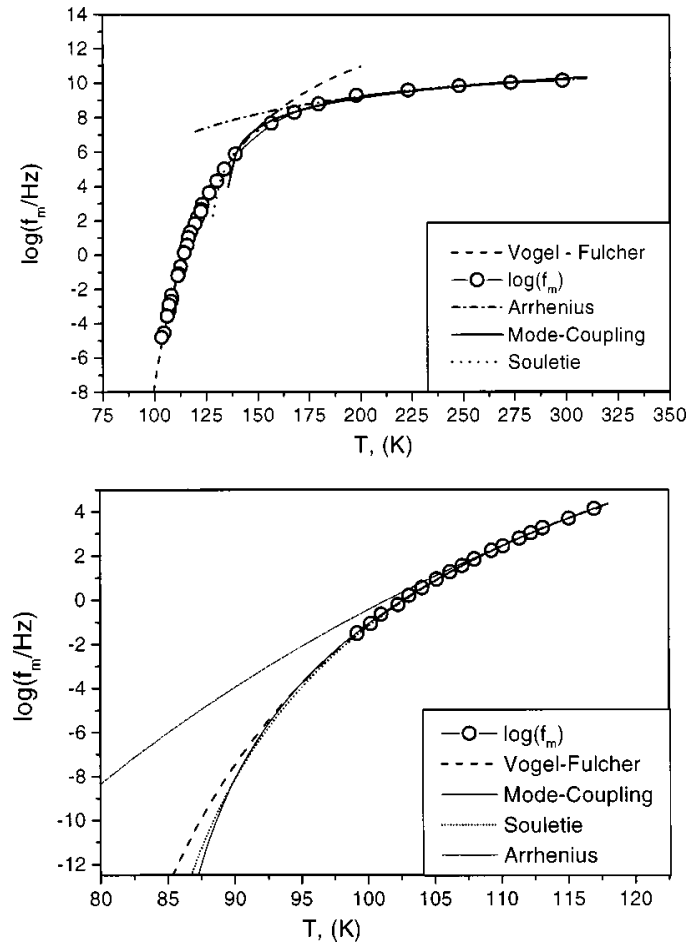

FIG. 9. The plots of $\log \left(f_{m}\right)$ against temperature in $\mathrm{K}$ for pure iso-amyl bromide (top), and $50 \mathrm{~mol} \%$ iso-amyl bromide solution in 2-methylpentane (bottom); and the calculated plots for the equations given by Arrhenius, Vogel, Fulcher, and Tamman, and by Souletie and that deduced from the mode-coupling theory, with parameters given in Table III.

the relaxation rates of supercooled liquids. This analysis is shown in Table III and Fig. 9 (top). The corresponding analysis of $\log \left(f_{m}\right)$ data for $50 \mathrm{~mol} \%$ iso-amyl bromide solution in 2-methyl pentane are also shown in Fig. 9 (bottom).

\section{The Johari-Goldstein relaxation}

One of the characteristic of supercooled liquid and glasses now generally recognized, and one on which the mode-coupling theory was originally based, is the faster relaxation process, which has also been seen as a precursor of the $\alpha$-relaxation process. This is known as the JohariGoldstein relaxation. The presence of a high frequency process was noted for pure iso-amyl bromide, ${ }^{25}$ but not detected for its solutions in 2-methylpentane. ${ }^{27}$ It is now clearly resolved for iso-amyl bromide in Fig. 2 and its spectral shape has been described by the $\mathrm{H}-\mathrm{N}$ equation with parameters given in Table I. However, only its presence has been detected in the iso-amyl bromide solution in 2-methylpentane, as shown in Fig. 7, but its amplitude appears to be too small to define unambiguously its spectral shape and rate. For the iso-amyl bromide, it follows the Arrhenius equation and appears to merge into the $\alpha$-relaxation process just near the temperature where Arrhenius-type dependence of $f_{m}$ changes to a Vogel-Fulcher-Tamman-type dependence. This suggests evolution of the $\alpha$-relaxation from the JohariGoldstein relaxation process at a temperature where the deviations begin to appear. Obviously, more liquids dissolved in nonpolar solvents need to be examined to verify it, but it is clear that the relaxation rate data for iso-amyl bromide agree with the finding by Hansen et al. ${ }^{5}$ and Stickel et al. ${ }^{8}$ regarding a change in the temperature dependence of the rate on supercooling a liquid.

\section{CONCLUSIONS}

The molecular dynamics in supercooled iso-amyl bromide occurs by two processes. The prominent, slower process is described by the Davidson-Cole relaxation function in which the distribution of relaxation time has a cutoff at a certain value. The weaker and faster relaxation process is described by a combination of the Cole-Cole and Davidson-Cole relaxation functions, in which the distribution of relaxation times is asymmetric, and the dielectric loss for the high frequency wing varies as $\omega^{-\alpha \beta}$. The JohariGoldstein process is thus found to be asymmetric. When the internal field effects are reduced by dissolving iso-amyl bromide in 2-methylpentane, the spectral shape of the prominent relaxation changes and becomes similar to that of the weak and the faster relaxation process. This change in the spectral shape is attributed to the decrease in dipolar interactions, and increase in steric hindrance evidence for 2-methylpentane being loosely packed compared to iso-amyl bromide which produces a polymer like dielectric spectra in solutions. The Johari-Goldstein relaxation occurs also in the solution but its amplitude is much reduced, and therefore remains undefined.

\section{ACKNOWLEDGMENTS}

The research was funded by the American Petroleum Research Fund PRF\# 33221-AC7 administered by the American Chemical Society and we acknowledge our thanks. We are grateful to Professor G. P. Johari for many stimulating discussions during the course of the writing of this paper, and to Dr. S. Havriliak Jr. for suggesting us to initiate this work. We thank Professor J. G. Berberian for providing his data on pure iso-amyl bromide along with the listing of the data from Dr. D. J. Denney and Dr. S. H. Glarum, Table I.

${ }^{1}$ Disorder Effects on Relaxational Processes, edited by R. Richert and A. Blumen (Springer-Verlag, Berlin, 1994).

${ }^{2}$ J. Jäckle, Rep. Prog. Phys. 49, 171 (1986).

${ }^{3}$ See Conference Proceedings issue of J. Non-Cryst. Solids 131-133, (1991), 172-174, (1994); 235-237, (1998).

${ }^{4}$ R. V. Chamberlin, Phys. Rev. B 48, 15638 (1993).

${ }^{5}$ C. Hansen, F. Stickel, T. Berger, R. Richert, and E. W. Fischer, J. Chem. Phys. 107, 1086 (1997), and references therein.

${ }^{6}$ E. Donth, Relaxation and Thermodynamics of Polymers (Hanser, Munich, 1992).

${ }^{7}$ K. L. Ngai, J. Chem. Phys. 109, 6982 (1998).

${ }^{8}$ F. Stickel, E. W. Fischer, and R. Richert, J. Chem. Phys. 102, 6251 (1995); 104, 2043 (1996), and references therein.

${ }^{9}$ A. Schönhals, F. Kremer, A. Hofmann, and E. W. Fischer, Physica A 201, 263 (1993).

${ }^{10}$ R. Kohlrausch, Pogg. Annal. Phys. 91, 179 (1854).

${ }^{11} \mathrm{R}$. Douglass, in Proceedings of the 4th International Congress on Rheology, Providence, RI, 1963, edited by E. H. Lee and A. L. Copley (Wiley, New York, 1965), Part 1, pp. 3-27; Br. J. Appl. Phys. 17, 435 (1966).

${ }^{12}$ G. Williams and D. C. Watts, Trans. Faraday Soc. 66, 80 (1970).

${ }^{13}$ S. Havriliak, Jr. and S. Negami, Polymer 8, 161 (1967); J. Polym. Sci., Part C: Polym. Symp. 14, 89 (1966).

${ }^{14}$ R. H. Cole and K. S. Cole, J. Chem. Phys. 9, 341 (1941).

${ }^{15}$ R. H. Cole, J. Chem. Phys. 23, 493 (1955). 
${ }^{16}$ D. W. Davidson and R. H. Cole, J. Chem. Phys. 18, 1417 (1950); 19, 1484 (1951).

${ }^{17}$ M. Menon, K. P. O'Brien, P. K. Dixon, L. Wu, S. R. Nagel, B. D. Williams, and J. P. Carini, J. Non-Cryst. Solids 141, 61 (1992).

${ }^{18}$ P. Lunkenheimer, A. Pimenov, B. Scheiner, R. Böhmer, and A. Loidl, Europhys. Lett. 33, 611 (1996).

${ }^{19}$ P. Lunkenheimer, A. Pimenov, M. Dressel, Yu. G. Goncharov, R. Böhmer, and A. Loidl, Phys. Rev. Lett. 77, 318 (1996).

${ }^{20}$ E. Rössler, U. Warschewske, P. Eirmann, A. P. Sokolov, and D. Quitmann, J. Non-Cryst. Solids 172-174, 113 (1994).

${ }^{21}$ A. Hofmann, F. Kremer, E. W. Fischer, and A. Schönhals, in Disorder Effects on Relaxational Processes, edited by R. Richert and A. Blumens (Springer-Verlag, Berlin, 1994).

${ }^{22}$ G. P. Johari and M. Goldstein, J. Chem. Phys. 53, 2372 (1970).
${ }^{23}$ G. P. Johari, J. Chem. Phys. 58, 1766 (1973).

${ }^{24}$ S. H. Glarum, J. Chem. Phys. 33, 639 (1960).

${ }^{25}$ D. J. Denney, J. Chem. Phys. 27, 259 (1957).

${ }^{26}$ J. G. Berberian and R. H. Cole, J. Am. Chem. Soc. 90, 3100 (1968).

${ }^{27}$ D. J. Denney and J. W. Ring, J. Chem. Phys. 44, 4621 (1966).

${ }^{28}$ H. Vogel, Phys. Z. 22, 645 (1921).

${ }^{29}$ G. S. Fulcher, J. Am. Ceram. Soc. 8, 339 (1923).

${ }^{30}$ G. Tammann and W. Hesse, Z. Anorg. Allg. Chem. 156, 245 (1926).

${ }^{31}$ G. P. Johari, J. G. McAnanama, and D. A. Wasylyshyn, J. Chem. Phys. 105, 10621 (1996) (see Appendix).

${ }^{32}$ M. G. Parthun and G. P. Johari, J. Chem. Soc., Faraday Trans. 91, 329 (1995).

${ }^{33}$ W. Götze and L. Sjögren, Rep. Prog. Phys. 55, 241 (1992).

${ }^{34}$ J. Soultie and D. Bertrand, J. Phys. I 1, 1627 (1991). 